\title{
TAGUNG
}

\section{Der Rat der Europäischen Union - Rolle und Reformen}

\author{
Tanja Leppik*
}

Der Rat der Europäischen Union nimmt eine zentrale Stellung im System der Europäischen Union ein und erfüllt mehrere Funktionen. Er ist zusammen mit dem Europäischen Parlament das gesetzgebende Organ und bildet gleichzeitig ein Forum für die Mitgliedstaaten, in dem sie ihre nationalen Interessen äuBern können. Dementsprechend finden sich unterschiedliche Sichtweisen hinsichtlich der Rolle des Rates. Diese ist im Institutionengefüge der Europäischen Union zudem nicht statisch, sondern hat sich auf Grund von Reformen des Ratssystems und der fortschreitenden Parlamentarisierung im Laufe der Jahre immer wieder verändert. Vor diesem Hintergrund gab die Tagung eine Bestandsaufnahme zur Entwicklung der Rolle des Rates im Institutionengefüge der Europäischen Union sowie seiner Arbeitsweise.

Auswirkungen der Ratsreformen auf das institutionelle Gleichgewicht

Im ersten Panel standen die Reformen des Ratssystems sowie die Veränderungen durch die Erweiterung und den Vertrag über eine Verfassung für Europa (VVE) im Mittelpunkt. Als Auftakt stellte Andreas Maurer die Organisationsreformen des Rates vor. Er wies darauf hin, dass auf Grund der Geschäftsautonomie des Rates Reformen auch ohne Vertragsänderungen möglich seien. So hatte der Europäische Rat 2002 in Sevilla beschlossen, getrennte Tagesordnungen für den koordinierenden und den außenpolitischen Teil des Rates für , Allgemeine Angelegenheiten und $\mathrm{Au}$ Benbeziehungen“ (RAA) einzuführen und die Anzahl der Fachratsformationen von 16 auf neun zu reduzieren, um die Arbeit des Rates

\section{Europapolitik im System des Rates der Europäischen Union}

Gemeinsame Fachtagung des Arbeitskreises Europäische Integration (AEI) und der Stiftung Wissenschaft und Politik (SWP)

Berlin, 20. Juni 2005

\section{Begrüßung und Einführung}

Dr. Andreas MAURER, SWP, Berlin

Panel I: Der Rat im Institutionengefüge der Union

Vorsitz: Dr. Andreas MAURER

Die Organisationsreformen des Rates

Dr. Andreas MAURER

Die Entwicklung des Ratssystems aus der Sicht des Auswärtigen Amtes

Dr. Nikolaus MEYER-LANDRUT, VLR I,

Europaabteilung, Auswärtiges Amt, Berlin

Die Entwicklung des Ratssystems aus der Sicht des Europäischen Parlaments

Dr. Wilhelm LEHMANN, Europäisches Parlament, Brüssel

Panel II: Funktionen und Rollenprofile des Rates

Vorsitz: Dr. Matthias JOPP, Institut für Europäische Politik, Berlin

Abstimmungsverhalten im Ministerrat

Wim VAN AKEN, EUI, Florenz

Die Ratsarbeitsgruppen

Prof. Dr. Jarle TRONDAL, Agder University College, Kristiansand

Der künftige Präsident des Europäischen Rates

Lars HOFFMANN, Universität Oxford Aufgaben und Profile des Ausschusses der Ständigen Vertreter Thorsten AUGUSTIN, Bundeskanzleramt

Tanja Leppik, Praktikantin in der Forschungsgruppe EU-Integration, Stiftung Wissenschaft und Politik, Berlin. 
effizienter zu gestalten. Außerdem wurden die impulsgebenden Funktionen des RAA und der Präsidentschaft dahingehend gestärkt, dass der Europäische Rat seit Dezember 2003 dreijährige Strategieprogramme erstellt und die zwei Präsidentschaften eines Jahres ein gemeinsames Arbeitsprogramm verabschieden.

Im VVE werden die Zusammensetzungen des Rates als Rat ,Allgemeine Angelegenheiten und als Rat ,Auswärtige Beziehungen' vertraglich festgelegt. Der Vorsitz im Rat soll nach einem ,System der gleichberechtigten Rotation ' erfolgen. Näheres soll ein Beschluss des Europäischen Rates regeln. Der derzeitige Entwurf dieses Beschlusses sieht einen Gruppenvorsitz von drei Mitgliedstaaten für einen Zeitraum von 18 Monaten vor. Ausgenommen von diesem Vorsitzsystem ist der Rat ,Auswärtige Angelegenheiten'. In dieser Zusammensetzung soll der neu eingeführte AuBenminister der Union den Vorsitz führen. Maurer erörterte die Frage, welche dieser Änderungen auch bei einem Scheitern des VVE realisiert werden könnten. Seiner Meinung nach wäre die Aufspaltung des RAA möglich, da sie nur die bereits existierende Praxis fortschreiben würde, dagegen erschiene die Änderung des sechsmonatigen Vorsitzes auf Ministerebene schwierig.

Die Reduzierung der Fachratsformationen hat nach Nikolaus Meyer-Landrut in der Praxis noch keine große Wirkung gezeigt. Auch das dreijährige Strategieprogramm spiele gegenüber dem Arbeitsprogramm der Kommission bisher nur eine untergeordnete Rolle. Dagegen habe die Erweiterung die Arbeit im Rat qualitativ geändert. Durch die gewachsene Zahl von Teilnehmern würden echte Verhandlungen erschwert. Deshalb läge mehr Gewicht auf den Tischumfragen, die ein generelles Stimmungsbild erzeugen könnten. Hintergründe und Positionen würden dagegen zunehmend von der Präsidentschaft in Einzelgesprächen mit den Mitgliedstaaten geklärt. Meyer-Landrut verwies zudem auf die große Bedeutung der vorbereitenden Gremien, wie
Panel 3: Der Rat in zentralen Politikfeldern der EU

Vorsitz: Dr. des. Daniela SCHWARZER, SWP, Berlin

\section{Der ECOFIN Rat}

Dr. Wolfgang GLOMB, Ständige Vertretung, Brüssel

Der Rat im Lissabon-Prozess

Peter BECKER, SWP, Berlin

Der Rat in der Justiz- und Innenpolitik

Dr. Andreas MAURER

Der Rat in den Auswärtigen Beziehungen

Dr. Daniel THYM, Walter Hallstein-Institut, Berlin

dem Ausschuss der Ständigen Vertreter (AStV) und den Arbeitsgruppen. In diesen Gremien würden bereits 80 bis 90 Prozent der Aspekte verhandelt. So kämen auf die Tagesordnung des Rates häufig so genannte APunkte, die von den Ministern nur noch zur Kenntnis genommen werden müssten.

Auch Thorsten Augustin betonte die wachsende Bedeutung des AStV. So habe der AStV etwa die Vorbereitung der Schlussfolgerungen für den Europäischen Rat übernommen, eine Aufgabe, die die Staats- und Regierungschefs früher selbst erledigt hätten. Zudem gebe der AStV häufig die entscheidenden Weichenstellungen und bereite die Sitzungen der verschiedenen Ratsformationen vor. Die Sitzungen für den RAA, den ECOFIN Rat und den Rat ,Justiz und Inneres “ werden vom AStV 2, der sich aus den Botschaftern der Ständigen Vertretungen zusammensetzt, vorbereitet. Der AStV 1, der aus den stellvertretenden Botschaftern besteht, bereitet die Tagungen der anderen Ratsformationen vor. Für Deutschland spiele nach Augustin der AStV 1 die größere Rolle, da in diesem Gremium viele Richtlinien und Verordnungen, die den politischen Alltag betreffen, ausgehandelt werden.

Doch nicht nur die Arbeitsweise des Rates, sondern auch seine Zusammenarbeit mit an- 
deren Institutionen hat sich geändert. Der Wandel in den Beziehungen zwischen Rat und Europäischem Parlament wurde von Wilhelm Lehmann erläutert. Seit der Einführung des Mitentscheidungsverfahrens hätten sich die Kontakte zwischen Rat und Europäischem Parlament stark intensiviert. So sprächen die Minister des Landes, das die Präsidentschaft innehat sowohl am Anfang als auch am Ende der Präsidentschaft in den Fachausschüssen des Europäischen Parlaments. Ausschussvorsitzende würden zunehmend $\mathrm{zu}$ informellen Treffen des Rates eingeladen. Einladungen der Ausschüsse würden von Ministern und anderen Ratsvertretern allerdings nur zögerlich wahrgenommen. In der Interinstitutionellen Vereinbarung ,Bessere Rechtssetzung von 2003 hätten Rat, Europäisches Parlament und Kommission zudem festgehalten, dass sie zur Erreichung einer besseren Rechtssetzung ihre Zusammenarbeit besser koordinieren wollten.

Die Arbeitsweise des Rates: Abstimmungen, vorbereitende Gremien und Vorsitz

Im zweiten Panel wurden verschiedene Aspekte der Arbeitsweise des Rates aus wissenschaftlicher Sicht beleuchtet. In der Literatur, aber auch in den Reformdiskussionen geht es dabei häufig um die Abstimmungsregeln und die Zusammensetzung der qualifizierten Mehrheit. Umfangreiches Material zum Abstimmungsverhalten im Rat für den Zeitraum von 1994 bis 2004 wird im Rahmen eines Projektes zusammengestellt, das Wim van Aken präsentierte. Auf der Basis der monatlichen Aufstellungen der Rechtsakte des Rates und der Abstimmungsprotokolle und Presseerklärungen zu den Ratssitzungen sowie weiterer Quellen werden Datensätze zum Abstimmungsverhalten im Rat erstellt. Anhand dieser Daten lässt sich sowohl der Anteil von Abstimmungen mit qualifizierter Mehrheit, bei denen es Enthaltungen und Gegenstimmen gab, als auch das Abstimmungsverhalten der einzelnen Mitgliedstaaten sowie das Abstimmungsverhalten in einzelnen Politikbereichen ermitteln. Van Aken erläuterte erste Er- kenntnisse bezüglich der Frage, wann und warum es im Rat zu abweichenden Voten komme. Die Daten zeigten, dass in etwa 25 Prozent der Entscheidungen mit qualifizierter Mehrheit abweichende Voten abgegeben würden. Die abweichenden Voten würden vor allem die Politikbereiche Landwirtschaft, Fischerei und Binnenmarkt betreffen. Hinsichtlich des Abstimmungsverhaltens einzelner Mitgliedstaaten habe sich gezeigt, dass Dänemark, Deutschland, die Niederlande, Großbritannien und auch Italien am häufigsten abweichende Voten abgeben würden, während Frankreich und Spanien weitaus seltener als andere große Mitgliedstaaten Gegenstimmen beziehungsweise Enthaltungen verwendeten. Die abweichenden Voten schienen mit spezifischen nationalen Interessen und innenpolitischen Gegebenheiten zusammenzuhängen. Koalitionsbildungen bestimmter Mitgliedstaaten, wie etwa große gegen kleine Mitgliedstaaten, ließen sich dagegen bei ersten Clusteranalysen nicht eindeutig erkennen.

Die Sichtweise vom Ministerrat als rein intergouvernementale Institution wurde von Jarle Trondal hinterfragt. Seine Studie zum Verhalten von nationalen Beamten in den Ratsarbeitsgruppen habe gezeigt, dass sich zwar viele nationale Beamte primär als nationale Repräsentanten sähen, dass aber einige Mitglieder der Ständigen Vertretungen zusätzlich eine verstärkte Rollenwahrnehmung als supranationaler Akteur aufwiesen. Diese tendenziell stärkere supranationale Ausrichtung der Mitglieder der Ständigen Vertretungen sei auf ihre ständige Präsenz vor Ort sowie auf ihre vielfältigen und intensiven formellen und informellen Kontakte innerhalb und außerhalb der Ratsarbeitsgruppen zurückzuführen. Dadurch könne es zu einer „Sekundärsozialisierung" der nationalen Beamten auf europäischer Ebene kommen. Demnach seien die Ratsarbeitsgruppen vorrangig als intergouvernementale Institutionen zu betrachten, die allerdings auch eine supranationale Komponente besäßen. 
Um die Kontinuität und die Sichtbarkeit der Ratsarbeit zu verbessern, wurde seit längerem über mögliche Reformen des Vorsitzsystems nachgedacht. Erste Überlegungen zu der Einführung eines ständigen Präsidenten für den Europäischen Rat wurden von Aznar, Blair und Chirac im so genannten ABC-Vorschlag im Frühjahr 2002 geäußert und durch den deutsch-französischen Vorschlag Anfang 2003 bekräftigt. In seinem Vortrag zeichnete Lars Hoffmann die Debatten im Konvent um die Einsetzung eines ständigen Präsidenten des Europäischen Rates nach. Im Konvent habe das Thema viel Zeit in Anspruch genommen, da die großen Mitgliedstaaten für die Einführung eines solchen Präsidenten plädiert hätten, um die Effizienz, Sichtbarkeit und Kontinuität zu verbessern, während sich die kleineren Mitgliedstaaten und die Kommission vehement dagegen ausgesprochen hätten, da sie eine Stärkung des Europäischen Rates auf Kosten der Kommission befürchteten. Hoffmann äußerte sich eher skeptisch über den vom VVE vorgesehenen hauptamtlichen Präsidenten des Europäischen Rates, der vom Europäischen Rat mit qualifizierter Mehrheit für eine zweieinhalbjährige Amtszeit gewählt werden soll. Laut Hoffmann könnte dieser Präsident das institutionelle Gleichgewicht sowie die gleichberechtigte Repräsentation der Mitgliedstaaten durch ihre Staats- und Regierungschefs im Europäischen Rat stören. Deshalb befürwortete Hoffmann das derzeitige System des rotierenden Vorsitzes.

\section{Die Rolle des Rates in verschiedenen Unions- politiken}

Im dritten Panel wurde die Funktion des Rates in einigen zentralen Politikbereichen dargestellt. Im Bereich Wirtschaft und Finanzen treffen sich die Wirtschafts- und Finanzminister der Mitgliedstaaten im ECOFIN Rat. Sie befassen sich mit vielfältigen Themen wie der Koordinierung der Wirtschaft oder der Haushaltspolitik. Die Mitgliedstaaten, die den Euro eingeführt haben, beraten sich zudem in der Eurogruppe, die meist am Vorabend der Sitzungen des ECOFIN Rates zusammentritt.
Wolfgang Glomb erläuterte aus Sicht des Praktikers die Arbeitsweise dieser Ratsformation sowie die Vorbereitungen durch den AStV 2 und den Wirtschafts- und Finanzausschuss. Hinsichtlich der Erweiterung stellte Glomb fest, dass die Arbeit des ECOFIN Rates sich zwar verändert habe, aber nicht schwieriger geworden sei. Er wies darauf hin, dass Entscheidungsverfahren häufig sehr komplex seien, da viele Rechtsakte im Mitentscheidungsverfahren beschlossen würden und es viele informelle Gespräche zwischen den Institutionen gäbe.

Einen weiteren zentralen Bereich stellt die Umsetzung der Lissabon-Strategie dar. Diese soll die Union ,zum wettbewerbsfähigsten und dynamischsten wissensbasierten Wirtschaftsraum der Welt" machen und berührt die Bereiche Wirtschaft, Beschäftigung, sozialer Zusammenhalt und Umwelt. Nach Peter Becker sei die „Lissabon-Methodik“ insgesamt als eine Mischform von ,hard" und ,soft politics' anzusehen. Denn sie beinhalte neben Rechtssetzungsverfahren nach der Gemeinschaftsmethode die Methode der offenen Koordinierung. Die Zieldefinition im Rahmen dieser Koordinierungsmethode werde von den Mitgliedstaaten und dem Rat dominiert. Eine zentrale Rolle spiele zudem der Europäische Rat, der auf seinen Frühjahresgipfeln eine leitende und koordinierende Funktion wahrnehme.

Ein relativ neues Politikfeld auf europäischer Ebene stellt die Innen- und Justizpolitik dar, die seit dem Vertrag von Amsterdam sowohl in der ersten als auch in der dritten Säule geregelt ist. Nach Andreas Maurer zeichnet sich dieses Politikfeld durch eine sehr dynamische Entwicklung aus: So ist die Anzahl der verabschiedeten Rechtsakte sowie der Anteil an verbindlichen Beschlüssen in den letzten zehn Jahren stark gestiegen. Mit Blick auf die Effizienz der Ratsarbeit stellte Maurer fest, dass die Entscheidungsverfahren auf Grund der Einstimmigkeit im Rat teilweise sehr langwierig und nicht sehr effizient gewesen seien. Zudem seien die Mitwirkungsmöglichkeiten 
der Kommission und des Europäischen Parlaments sehr beschränkt. Die geringe Beteiligung des Europäischen Parlaments ist nach Maurer vor allem mit Blick auf Demokratiestandards von Bedeutung. Im Haager Programm werde über die Einführung des Mitentscheidungsverfahrens und damit über eine stärkere Beteiligung des Europäischen Parlaments nachgedacht. Allerdings obliege es dem Rat, einen entsprechenden Beschluss zu verabschieden. Wichtig sei nach Maurer auch eine aktivere Rolle der nationalen Parlamente.

Der Begriff Außenbeziehungen umfasst Bereiche wie Entwicklungspolitik, Außenhandel und Gemeinsame Außen- und Sicherheitspolitik. Entsprechend der Zuordnung dieser Politiken auf die erste und zweite Säule kommt dabei entweder die Gemeinschaftsmethode oder die Unionsmethode zur Anwendung. Daniel Thym befasste sich in seinem Vortrag mit der Rolle des Rates sowie mit aktuellen Entwicklungstendenzen des ministerialbürokratischen Unterbaus in diesen Bereichen. Zudem erörterte er aus rechtlicher Sicht die Umsetzungsmöglichkeiten von einigen Reformvorschlägen des VVE zu den Außenbeziehungen. Vertraglich geregelte Dinge wie die Ausweitung des Mitentscheidungsverfahrens bedürften einer Vertragsänderung. Intrainstitutionelle Reformen wie die Einsetzung eines ständigen Präsidenten für den Europäischen Rat könnten dagegen weitgehend um- gesetzt werden. Die Einführung des Außenministers und des Europäischen Auswärtigen Dienstes würde allerdings das institutionelle Gleichgewicht stören und erforderte deshalb eine Vertragsänderung.

\section{Fazit}

Die Beiträge und Diskussionen machten deutlich, dass die Ausdehnung des Mitentscheidungsverfahrens und die Ratsreformen zu Verschiebungen im institutionellen Gleichgewicht geführt haben und dass die Erweiterung die internen Entscheidungsabläufe des Rates verändert hat. Hinsichtlich der Auswirkungen der Ratsreformen im VVE auf das institutionelle Gleichgewicht wurde festgestellt, dass die Einführung eines hauptamtlichen Präsidenten des Europäischen Rates sowie die Ausgestaltung seines Verhältnisses zu dem rotierenden Vorsitz der Ratsformationen, dem Kommissionspräsidenten und dem Außenminister größere Auswirkungen haben werde als die geplanten Teampräsidentschaften. Für die Handlungsfähigkeit des Rates wurde die vorgesehene Ausweitung der Anwendungsbereiche von Abstimmungen mit qualifizierter Mehrheit als besonders wichtig erachtet. Aufgabe der Wissenschaft sei es, Strategien zu entwickeln für den Fall, dass der VVE nicht von allen Mitgliedstaaten ratifiziert werden sollte. 